\title{
Concentrated solutions for a critical nonlocal problem
}

\section{Qingfang Wang ${ }^{1 *}$ (D)}

"Correspondence:

hbwangqingfang@163.com

${ }^{1}$ School of Mathematics and Computer Science, Wuhan

Polytechnic University, Wuhan, 430023, P.R. China

\section{Springer}

\begin{abstract}
In this paper, we deal with a class of fractional critical problems. Under some suitable assumptions, we derive the existence of a positive solution concentrating at the critical point of the Robin function by using the Lyapunov-Schmidt reduction method. Comparing with previous work, we encounter some new challenges because of a nonlocal term. By making some delicate estimates for the nonlocal term we overcome the difficulty and find a bubbling solution.
\end{abstract}

MSC: 35B33; 35J60

Keywords: Fractional Laplacian; Lyapunov-Schmidt reduction method; Critical nonlinearity

\section{Introduction}

This paper is concerned with the solution for the following elliptic equation involving fractional spectral Laplacian and critical exponent:

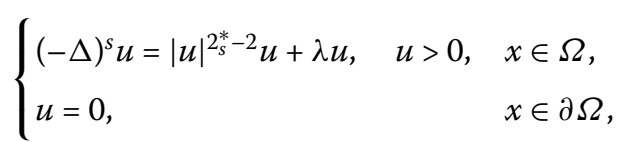

where $0<s<1,2_{s}^{*}=\frac{2 N}{N-2 s}, \Omega$ is a smooth bounded domain of $\mathbb{R}^{N}$. $(-\Delta)^{s}$ denotes the fractional Laplace operator, and $\lambda_{1}(\Omega)$ is the first eigenvalue of $(-\Delta)^{s}$ in $\Omega$ under zero Dirichlet boundary data.

The fractional power of the Laplacian $(-\Delta)^{s}$ appears in diverse areas including physic, biological modeling and mathematical finances; see $[6,7,12]$. An important quality of the fractional Laplacian is its nonlocal property, which makes it difficult to handle. Caffarelli and Silvestre gave a new method which allows one to transform nonlocal problems to local ones in [8]. Many researchers studied nonlinear problems of Eq. (1.1) based on these extensions which permit it to use variational methods. More precisely, for the subcritical exponent, Dipierro et al. proved the existence of a positive and spherically symmetric solution in [13]. Recently, Wang and Zhou [27] also considered subcritical case, they obtained the existence of a radial sign-changing solution by using Brouwer degree theory and variational method. In [30], Yan et al. obtain infinitely many solutions as an application of the

(c) The Author(s) 2020. This article is licensed under a Creative Commons Attribution 4.0 International License, which permits use sharing, adaptation, distribution and reproduction in any medium or format, as long as you give appropriate credit to the original author(s) and the source, provide a link to the Creative Commons licence, and indicate if changes were made. The images or other third party material in this article are included in the article's Creative Commons licence, unless indicated otherwise in a credit line to the material. If material is not included in the article's Creative Commons licence and your intended use is not permitted by statutory regulation or exceeds the permitted use, you will need to obtain permission directly from the copyright holder. To view a copy of this licence, visit http://creativecommons.org/licenses/by/4.0/. 
compactness result. For the equation $(-\Delta)^{s}=u^{q}$ with the supercritical exponents $q \geq \frac{N+2 s}{N-2 s}$, the nonexistence of solutions was proved in $[3,25,26]$ in which one used the Pohozaev type identities. Partial differential equations involving the fractional Laplacian have attracted the attention of many researchers; see for example $[2,5,6,8,14,15,17,18,22,23,29]$ and the references therein.

The analogue problem to (1.1) for the Laplacian operator has been studied extensively in recent years; see $[1,4,6,11,28]$ and the references therein. For $s=1$, the equation becomes the Brezis-Nirenberg problem

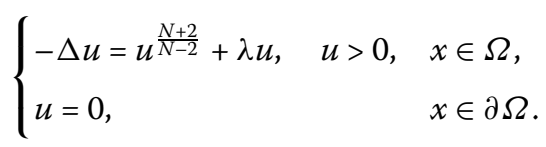

Rey [20] constructed a family of solutions which asymptotically blow up at a nondegenerate critical point of the Robin function. Moreover, this result was extended in [19], where Musso and Pistoia obtained the existence of multi-peak solutions for certain domains. In [4], Brezis and Nirenberg considered the existence of positive solutions for problem (1.1) with $s=1$. It is well known that the Sobolev embedding $H_{0}^{1}(\Omega) \hookrightarrow L^{2^{*}}(\Omega)$ is not compact even if $\Omega$ is bounded. In [16], a concentration-compactness principle was developed to treat non-compact critical variational problems. A global compactness result was found in [24] which describes precisely the obstacles of the compactness for critical semilinear elliptic problems.

The aim of this paper is to study the problem when $p=\frac{N+2 s}{N-2 s}$ is the critical Sobolev exponent and $\lambda>0$ is close to zero. Using variational methods and Lyapunov-Schmidt reduction, we prove that Eq. (1.1) admits a positive solution concentrating at the critical point of the Robin function. However, due to the fact that the fractional Laplacian operator is nonlocal, very few things on this topic are known about the fractional Laplacian. We point out that we adopt in the paper the spectral definition of the fractional Laplacian in a bounded case with a Caffarelli-Silverstre type extension [9], and not the integral definition. We refer to [21] for a nice comparison between these two different notions.

We set the fractional Sobolev space $H_{0}^{s}(\Omega)(0<s<1)$ by

$$
H_{0}^{s}(\Omega)=\left\{u=\sum_{k=1}^{\infty} a_{k} \phi_{k} \in L^{2}(\Omega): \sum_{k=1}^{\infty} a_{k}^{2} \lambda_{k}^{s}<\infty\right\}
$$

which is a Hilbert space whose inner product is given by

$$
\left\langle\sum_{k=1}^{\infty} a_{k} \phi_{k}, \sum_{k=1}^{\infty} b_{k} \phi_{k}\right\rangle_{H_{0}^{s}(\Omega)}=\sum_{k=1}^{\infty} a_{k} b_{k} \lambda_{k}^{s} \quad \text { if } \sum_{k=1}^{\infty} a_{k} \phi_{k}, \sum_{k=1}^{\infty} b_{k} \phi_{k} \in H_{0}^{s}(\Omega) .
$$

Moreover, for a function in $H_{0}^{s}(\Omega)$, we define the fractional Laplacian as

$$
(-\Delta)^{s}\left(\sum_{k=1}^{\infty} a_{k} \phi_{k}\right)=\sum_{k=1}^{\infty} a_{k} \lambda_{k}^{s} \phi_{k}
$$


We also consider the square root $(-\Delta)^{\frac{s}{2}}: H_{0}^{s}(\Omega) \rightarrow L^{2}(\Omega)$. Note that by the above definitions, we have

$$
\langle u, v\rangle_{H_{0}^{s}(\Omega)}=\int_{\Omega}(-\Delta)^{\frac{s}{2}} u \cdot(-\Delta)^{\frac{s}{2}} v d x=\int_{\Omega}(-\Delta)^{s} u \cdot v d x \quad \text { for } u, v \in H_{0}^{s}(\Omega) .
$$

If the domain $\Omega$ is the whole space $\mathbb{R}^{N}$, the space $H^{s}\left(\mathbb{R}^{N}\right)(0<s<1)$ is given as

$$
H^{s}\left(\mathbb{R}^{N}\right)=\left\{u \in L^{2}\left(\mathbb{R}^{N}\right):\|u\|_{H^{s}\left(\mathbb{R}^{N}\right)}:=\left(\int_{\mathbb{R}^{N}}\left(1+|2 \pi \xi|^{2 s}\right)|\hat{u}(\xi)|^{2} d \xi\right)^{\frac{1}{2}}<\infty\right\},
$$

where $\hat{u}$ denotes the Fourier transform of $u$, and the fractional Laplacian $(-\Delta)^{s}$ is defined to be

$$
\widehat{(-\Delta)^{s}} u(\xi)=|2 \pi \xi|^{2 s} \hat{u}(\xi) .
$$

Definition 1.1 For a function $u \in H_{0}^{s}(\Omega)$, we denote its s-harmonic extension $w=E_{s}(u)$ to the cylinder $\mathcal{C}$ as the solution of the problem

$$
\begin{cases}\operatorname{div}\left(t^{1-2 s} \nabla w\right)=0, & \text { in } \mathcal{C}, \\ w=0, & \text { on } \partial_{L} \mathcal{C}, \\ w(x, 0)=u(x), & \text { on } x \in \Omega \times\{0\}\end{cases}
$$

and

$$
(-\Delta)^{s} u(x)=-k_{s} \lim _{y \rightarrow 0^{+}} y^{1-2 s} \frac{\partial w}{\partial y}(x, y),
$$

where $k_{s}=2^{1-2 s} \Gamma(1-s) / \Gamma(s)$ is a normalization constant.

The extension function $w(x, y)$ belongs to the space

$$
H_{0, L}^{1}(\mathcal{C}):={\overline{C_{0}^{\infty}(\Omega \times[0, \infty))}}^{\|\cdot\|_{H_{0, L}^{1}}(\mathcal{C})}
$$

endowed with the norm

$$
\|w\|_{H_{0, L}^{1}(\mathcal{C})}=\left(k_{s} \int_{\mathcal{C}} y^{1-2 s}|\nabla w|^{2} d x d y\right)^{\frac{1}{2}}
$$

The extension operator is an isometry between $H_{0}^{s}(\Omega)$ and $H_{0, L}^{1}(\mathcal{C})$, namely

$$
\|u\|_{H_{0}^{s}(\Omega)}=\left\|E_{s}(u)\right\|_{H_{0, L}^{1}(\mathcal{C})} \quad \text { for all } u \in H_{0}^{s}(\Omega) .
$$

With this definition, we see problem (1.1) is the Brezis-Nirenberg type problem with the fractional Laplacian. To treat the nonlocal problem (1.1), we shall study a corresponding extension problem; we refer the reader to $[2,3,22]$ and the references therein. Therefore, 
the nonlocal problem (1.1) can be reformulated to the following local problem:

$$
\begin{cases}\operatorname{div}\left(t^{1-2 s} \nabla v\right)=0, & \text { in } \mathcal{C}, \\ v=0, & \text { on } \partial_{L} \mathcal{C}, \\ \lim _{y \rightarrow 0^{+}} y^{1-2 s} \frac{\partial v}{\partial v}=|v(x, 0)|^{2_{s}^{*}-2} v(x, 0)+\lambda v(x, 0), & \text { on } \Omega \times\{0\}\end{cases}
$$

where $\frac{\partial}{\partial v}$ is the outward normal derivative of $\partial \mathcal{C}$. The extension operator is an isometry between $H_{0}^{s}(\Omega)$ and $H_{0, L}^{1}(\mathcal{C})$, namely

$$
\|u\|_{H_{0}^{s}(\Omega)}=\|v\|_{H_{0, L}^{1}(\mathcal{C})} \quad \text { for all } u \in H_{0}^{s}(\Omega) .
$$

Hence, critical points of the functional

$$
I(v)=\frac{1}{2 C_{s}} \int_{\mathcal{C}} y^{1-2 s}|\nabla v|^{2} d x d y-\frac{1}{2_{s}^{*}} \int_{\Omega \times\{0\}}|v|^{2_{s}^{*}} d x-\frac{\lambda}{2} \int_{\Omega \times\{0\}}|v|^{2} d x
$$

defined on $H_{0, L}^{1}(\mathcal{C})$ corresponding to solutions of (1.5). Without loss of generality, we may assume $C_{s}=1$.

Now we introduce the Green's function of $(-\Delta)^{s}$ with the Dirichlet boundary condition, which solves

$$
(-\Delta)^{s} G(\cdot, y)=\delta_{y} \quad \text { in } \Omega \quad \text { and } \quad G(\cdot, y)=0 \quad \text { on } \partial \Omega .
$$

The regular part of $G$ is given by

$$
H(x, y)=\frac{a_{N, s}}{|x-y|^{N-2 s}}-G(x, y) \quad \text { where } a_{N, s}=\frac{1}{\left|S^{N-1}\right|} \frac{2^{1-2 s} \Gamma\left(\frac{N-2 s}{2}\right)}{\Gamma\left(\frac{N}{2}\right) \Gamma(s)} .
$$

The diagonal part $\tau$ of the function $H$, namely, $\tau(x):=H(x, x)$ for $x \in \Omega$ is called the Robin function and it plays a crucial role for our problem.

Theorem 1.2 Assume $0<s<1$ and $N>4 s$, the equation has a bubbling solution which concentrated at the local minimum of the Robin function.

This paper is organized as follows. In Sect. 2, we study the regularity of the Green's function of fractional Laplacian and show some estimates. In Sect. 3, using the LyapunovSchmidt reduction method, we prove the main theorem. We exhibit some necessary computations for the construction of concentrating solutions in the appendix.

\section{Some preliminaries and estimates}

In the following lemma we list some relevant inequalities from [3].

Lemma 2.1 For any $1 \leq r \leq 2_{s}^{*}$ and any $z \in H_{0, L}^{1}(\mathcal{C})$, we have

$$
\left(\int_{\Omega}|u(x)|^{r} d x\right)^{\frac{2}{r}} \leq C \int_{\mathcal{C}} y^{1-2 s}|\nabla z(x, y)|^{2} d x d y, \quad u=\operatorname{Tr}(z)
$$

for some positive constant $C=C(r, s, N, \Omega)$. 
When $r=2_{s}^{*}$, the best constant in $(2.1)$ is denoted by $S(s, N)$, that is,

$$
S(s, N):=\inf _{z \in H_{0, L}^{1}(\mathcal{C}) \backslash\{0\}} \frac{\int_{\mathcal{C}} y^{1-2 s}|\nabla z(x, y)|^{2} d x d y}{\left(\int_{\Omega}|z(x, 0)|^{*} d x\right)^{\frac{2}{2_{s}^{*}}}},
$$

where $S(s, N)$ is achieved for $\Omega=\mathbb{R}^{N}$ by function $U_{x, \mu}$ which are the s-harmonic extension of $u_{x, \mu}$, where

$$
u_{x, \mu}=a_{N, s}\left(\frac{\mu}{1+\mu^{2}\left|x-x_{\lambda}\right|^{2}}\right)^{\frac{N-2 s}{2}} .
$$

Let $U(x)=\left(1+|x|^{2}\right)^{\frac{2 s-N}{2}}$ and let $\mathcal{W}$ be the extension of $U$. Then

$$
\mathcal{W}(x, y)=E_{s}(U)=c_{N, s} \int_{\mathbb{R}^{N}} \frac{U(z) d z}{\left(|x-z|^{2}+y^{2}\right)^{\frac{N+2 s}{2}}}
$$

is the extreme function for the fractional Sobolev inequality (2.2). The constant $\mathcal{S}(s, N)$ takes the exact value

$$
\mathcal{S}(s, N)=\frac{2 \pi^{s} \Gamma(1-s)\left(\Gamma\left(\frac{N}{2}\right)\right)^{\frac{2 s}{N}}}{\Gamma(s) \Gamma\left(\frac{N-2 s}{2}\right)(\Gamma(N))^{s}} .
$$

It was shown that, if a suitable decay assumption is imposed, then $\left\{u_{x, \mu}: \mu>0, x \in \mathbb{R}^{N}\right\}$ is the set of all solutions for the problem

$$
(-\Delta)^{s} u=u^{p}, \quad u>0 \text { in } \mathbb{R}^{N} \text { and } \quad \lim _{|x| \rightarrow \infty} u(x)=0 .
$$

We use $U_{x, \mu} \in D^{s}\left(\mathbb{R}_{+}^{N+1}\right)$ to denote the s-harmonic extension of $u_{x, \mu}$, so that $U_{x, \mu}$ solves

$$
\begin{cases}\operatorname{div}\left(t^{1-2 s} U_{x, \mu}(x, t)\right)=0, & (x, t) \in \mathbb{R}_{+}^{N+1}, \\ U_{x, \mu}(x, 0)=u_{x, \mu}(x), & x \in \mathbb{R}^{N} .\end{cases}
$$

Now we introduce the Green's function in case of s-harmonic extension operator. Let $G$ be the Green's function of the fractional Laplacian $(-\Delta)^{s}$ with the zero Dirichlet boundary condition. Then it can be regarded as the trace of the Green's function $G_{\mathcal{C}}=G_{\mathcal{C}}(z, x)$ for the extended Dirichlet-Neumann problem which satisfies

$$
\begin{cases}\operatorname{div}\left(t^{1-2 s} \nabla G_{\mathcal{C}}(\cdot, x)\right)=0, & \text { in } \mathcal{C}, \\ G_{\mathcal{C}}(\cdot, x)=0, & \text { on } \partial_{L} \mathcal{C}, \\ \partial_{\nu}^{s} G_{\mathcal{C}}(\cdot, x)=\delta_{x}, & \text { on } \Omega \times\{0\} .\end{cases}
$$

In fact, if a function $W$ in $\mathcal{C}$ solves

$$
\begin{cases}\operatorname{div}\left(t^{1-2 s} \nabla W\right)=0, & \text { in } \mathcal{C}, \\ W=0, & \text { on } \partial_{L} \mathcal{C}, \\ \partial_{v}^{s} W=g, & \text { on } \Omega \times\{0\}\end{cases}
$$


for some function $g$ on $\Omega \times\{0\}$, then we can see that $W$ has the expression

$$
W(z)=\int_{\Omega} G_{\mathcal{C}}(z, y) g(y) d y=\int_{\Omega} G_{\mathcal{C}}(z, y)(-\Delta)^{s} w(y) d y, \quad z \in \mathcal{C},
$$

where $w=\left.\operatorname{tr}\right|_{\Omega \times\{0\}} W$. Then, by plugging $z=(x, 0)$ in the above equalities, we obtain

$$
w(x)=\int_{\Omega} G_{\mathcal{C}}((x, 0), y)(-\Delta)^{s} w(y) d y,
$$

which implies that $G_{\mathcal{C}}((x, 0), y)=G(x, y)$ for any $x, y \in \Omega$.

The Green's function $G_{\mathcal{C}}$ on the half cylinder $\mathcal{C}$ can be partitioned to the singular part and regular part. The single part is

$$
G_{\mathbb{R}_{+}^{N+1}}((x, t), y):=\frac{a_{N, s}}{|(x-y, t)|^{N-2 s}},
$$

which satisfies

$$
\begin{cases}\operatorname{div}\left(t^{1-2 s} \nabla_{x, t} G_{\mathbb{R}_{+}^{N+1}}((x, t), y)=0,\right. & \text { in } \mathbb{R}_{+}^{N+1}, \\ \partial_{\nu}^{s} G_{\mathbb{R}_{+}^{N+1}}((x, 0), y)=\delta_{y}(x), & \text { on } \Omega \times\{0\} .\end{cases}
$$

In this section, we prove Theorem 1.2 by applying the Lyapunov-Schmidt reduction method to the extended problem

$$
\begin{cases}\operatorname{div}\left(t^{1-2 s} \nabla v\right)=0, & \text { in } \mathcal{C}=\Omega \times(0, \infty), \\ v=0, & \text { on } \partial_{L} \mathcal{C}=\partial \Omega \times(0, \infty), \\ \partial_{v}^{s} v=v^{p}+\lambda v, & \text { on } \Omega \times\{0\},\end{cases}
$$

where $0<s<1$ and $p=\frac{N+2 s}{N-2 s}$. We recall that the functions $u_{x, \mu}$ and $U_{x, \mu}$ are defined in (2.3). It is known that the space of the bounded solutions for the linearized equation

$$
(-\Delta)^{s} \phi=p u_{x, \mu}^{p-1} \phi \quad \text { in } \mathbb{R}^{N}
$$

is spanned by

$$
\frac{\partial u_{x, \mu}}{\partial x_{1}}, \ldots, \frac{\partial u_{x, \mu}}{\partial x_{N}} \text { and } \frac{\partial u_{x, \mu}}{\partial \mu},
$$

where $x=\left(x_{1}, \ldots, x_{N}\right)$ represents the variable in $\mathbb{R}^{N}$. By the results of Dávial, del Pino and Sire [10], it also follows that the solutions of the extended problem of

$$
\begin{cases}\operatorname{div}\left(t^{1-2 s} \nabla \Phi\right)=0, & \text { in } \mathbb{R}_{+}^{N+1}=\mathbb{R}^{N} \times(0,+\infty), \\ \partial_{\nu}^{s} \Phi=p U_{x, \mu}^{p-1} \Phi, & \text { on } \mathbb{R}^{N} \times\{0\},\end{cases}
$$

which are bounded on $\Omega \times\{0\}$, consist of the linear combinations of

$$
\frac{\partial U_{x, \mu}}{\partial x_{1}}, \ldots, \frac{\partial U_{x, \mu}}{\partial x_{N}}, \frac{\partial U_{x, \mu}}{\partial \mu} .
$$


We define $P_{\Omega}$ such that

$$
\begin{cases}\partial_{\nu}^{s}\left(P_{\Omega} U_{x, \mu}\right)=\partial_{v}^{s} U_{x, \mu}=U_{x, \mu}^{2_{s}^{*}-1}, & x \in \Omega \times\{0\} \\ P_{\Omega} U_{x, \mu}=0, & x \in \partial \Omega \times(0,+\infty) .\end{cases}
$$

Let $\varphi=U_{x, \mu}-P_{\Omega} U_{x, \mu}$, we have

$$
\begin{cases}\operatorname{div}\left(t^{1-2 s} \nabla \varphi\right)=0, & \text { in } \Omega \times(0,+\infty), \\ \left.\varphi\right|_{\partial \Omega}=\left.U_{x, \mu}\right|_{\partial \Omega}=\frac{C_{0}}{\mu^{\frac{N-2 s}{2}}\left|y-x_{\lambda}\right|^{N-2 s}}\left(1+O\left(\frac{1}{\mu^{2}\left|y-x_{\lambda}\right|^{2}}\right)\right), & \\ \partial_{v}^{s} \varphi=0, & \text { on } \Omega \times\{0\} .\end{cases}
$$

Consider

$$
\begin{cases}\operatorname{div}\left(t^{1-2 s} \nabla_{(x, t)} H_{\mathcal{C}}((x, t), y)\right)=0, & \text { in } \mathcal{C}, \\ \left.H_{\mathcal{C}}((x, t), y)\right)=\frac{\mathfrak{a}_{N, s}}{|(x-y), t|^{N-2 s}}, & \text { on } \partial_{L} \mathcal{C}, \\ \partial_{v}^{s} H_{\mathcal{C}}((x, 0), y)=0, & \text { on } \Omega \times\{0\},\end{cases}
$$

then $H_{\mathcal{C}}((x, t), y)$ is the regular part of the Green's function. Thus, we obtain

$$
U_{x, \mu}-P_{\Omega} U_{x, \mu}=\frac{C_{0}}{\mu^{\frac{N-2 s}{2}}} H((x, t), y) .
$$

We define

$$
E=\left\{U \in H_{0, L}^{1}(\mathcal{C}):\left\langle U, \frac{\partial U_{x, \mu}}{\partial x_{j}}\right\rangle_{H_{0, L}^{1}(\mathcal{C})}=0, j=0,1, \ldots, N\right\}
$$

In order to prove Theorem 1.2, we only need to prove the following proposition.

Proposition 2.2 Under the assumption of Theorem 1.2, (1.5) has a solution $v$ of the form

$$
\nu=P_{\Omega} U_{x, \mu}+\omega,
$$

where $\omega \in E, P_{\Omega} U_{x, \mu}$ defined in (2.11), $\|w\|_{H_{0, L}^{1}(\mathcal{C})} \rightarrow 0, x_{\lambda} \rightarrow x_{0}$ as $\lambda \rightarrow 0$.

The energy function corresponding to (1.5) is

$$
I(v):=\frac{1}{2} \int_{\mathcal{C}} y^{1-2 s}|\nabla v|^{2} d x d y-\frac{\lambda}{2} \int_{\Omega \times\{0\}}|v|^{2} d x-\frac{1}{p+1} \int_{\Omega \times\{0\}}|v|^{p+1} d x
$$

We expand $J(\omega)$ as follows:

$$
J(\omega)=I\left(P_{\Omega} U_{x, \mu}+\omega\right)=J(0)+\ell(\omega)+\frac{1}{2} L(\omega)+R(\omega),
$$


where

$$
\begin{aligned}
L(\omega)= & \int_{\mathcal{C}} y^{1-2 s}|\nabla \omega|^{2} d x d y-\lambda \int_{\Omega \times\{0\}} \omega^{2} d x \\
& -\left(2_{s}^{*}-1\right) \int_{\Omega \times\{0\}}\left|P_{\Omega} U_{x, \mu}\right|^{2_{s}^{*}-2} \omega^{2} d x, \\
\ell(\omega)= & \int_{\Omega \times\{0\}} U_{x, \mu}^{2_{s}^{*}-1} \omega d x d y-\int_{\Omega \times\{0\}}\left|P_{\Omega} U_{x, \mu}\right|^{2_{s}^{*}-1} \omega d x-\lambda \int_{\Omega \times\{0\}} P_{\Omega} U_{x, \mu} \omega d x,
\end{aligned}
$$

and

$$
\begin{aligned}
R(\omega)= & \frac{1}{2_{s}^{*}} \int_{\Omega \times\{0\}}\left(\left|P_{\Omega} U_{x, \mu}+\omega\right|^{2_{s}^{*}}-\left|P_{\Omega} U_{x, \mu}\right|^{2_{s}^{*}}-2_{s}^{*}\left|P_{\Omega} U_{x, \mu}\right|^{2_{s}^{*}-1} \omega\right. \\
& \left.-\left(2_{s}^{*}\right)\left(2_{s}^{*}-1\right)\left|P_{\Omega} U_{x, \mu}\right|^{2_{s}^{*}-2} \omega^{2}\right) d x
\end{aligned}
$$

is the higher order of $\omega$.

In order to find a critical point for $J(\omega)$, we need to discuss each term in the expansion (2.16). We will use $x$ instead of $x_{\lambda}$ for simplicity in this paper.

Now we arrive at the main result in this section.

Lemma 2.3 There is a constant $C>0$ independent of $\mu$ such that

$$
\left\|R^{\prime}(\omega)\right\|_{H_{0, L}^{1}(\mathcal{C})} \leq C\|\omega\|_{H_{0, L}^{1}(\mathcal{C})}^{\min \left\{\left\{_{-}^{*}-1,2\right\}\right.}
$$

and

$$
\left\|R^{\prime \prime}(\omega)\right\|_{H_{0, L}^{1}(\mathcal{C})} \leq C\|\omega\|_{H_{0, L}^{1}(\mathcal{C})}^{\min \left\{2^{*}-2,1\right\}} .
$$

Proof By a direct calculation, in the case $2_{s}^{*}-1>2$, we know that

$$
\begin{aligned}
\left\langle R^{\prime}(\omega), \phi\right\rangle & =\int_{\Omega \times\{0\}}\left(\left|P_{\Omega} U_{x, \mu}+\omega\right|^{2_{s}^{*}-1}-\left|P_{\Omega} U_{x, \mu}\right|^{2_{s}^{*}-1}-\left(2_{s}^{*}-1\right)\left|P_{\Omega} U_{x, \mu}\right|^{2_{s}^{*}-2} \omega\right) \phi d x \\
& \leq C \int_{\Omega \times\{0\}}\left|P_{\Omega} U_{x, \mu}\right|^{2_{s}^{*}-3} \omega^{2} \phi d x \\
& \leq C\left(\int_{\Omega \times\{0\}}\left(\left|P_{\Omega} U_{x, \mu}\right|^{2_{s}^{*}-3} \omega^{2}\right)^{\frac{2_{s}^{*}}{2_{s}^{*}-1}} d x\right)^{\frac{2_{s}^{*}-1}{2_{s}^{*}}}\left(\int_{\Omega \times\{0\}} \phi^{2_{s}^{*}} d x\right)^{\frac{1}{2_{s}^{*}}} \\
& \leq C\|\omega\|_{H_{0, L}^{1}(\mathcal{C})}^{2}\|\phi\|_{H_{0, L}^{1}(\mathcal{C})}
\end{aligned}
$$

and

$$
\begin{aligned}
\left\langle R^{\prime \prime}(\omega),(\phi, \psi)\right\rangle & =\int_{\Omega \times\{0\}}\left(\left|P_{\Omega} U_{x, \mu}+\omega\right|^{2_{s}^{*}-2}-\left|P_{\Omega} U_{x, \mu}\right|^{2_{s}^{*}-2}\right) \phi \psi d x \\
& \leq C \int_{\Omega \times\{0\}}\left(P_{\Omega} U_{x, \mu}\right)^{2_{s}^{*}-3} \omega \phi \psi d x \\
& \leq C\left(\int_{\Omega \times\{0\}}\left(\left|P_{\Omega} U_{x, \mu}\right|^{2_{s}^{*}-3} \omega \phi\right)^{\frac{2_{s}^{*}}{2_{s}^{*}-1}} d x\right)^{\frac{2_{s}^{*}-1}{2_{s}^{*}}}\left(\int_{\Omega \times\{0\}} \psi^{2_{s}^{*}} d x\right)^{\frac{1}{2_{s}^{*}}}
\end{aligned}
$$




$$
\leq C\|\omega\|_{H_{0, L}^{1}(\mathcal{C})}\|\phi\|_{H_{0, L}^{1}(\mathcal{C})}\|\psi\|_{H_{0, L}^{1}(\mathcal{C})}
$$

Now, we deal with $2_{s}^{*}-1<2$ to obtain

$$
\begin{aligned}
& \left\langle R^{\prime}(\omega), \phi\right\rangle \leq C \int_{\Omega \times\{0\}} \omega^{2_{s}^{*}-1} \phi d x \leq C\|\omega\|_{H_{0, L}^{1}(\mathcal{C})}^{2^{*}-1}\|\phi\|_{H_{0, L}^{1}(\mathcal{C})}, \\
& \left\langle R^{\prime \prime}(\omega),(\phi, \psi)\right\rangle \leq C \int_{\Omega \times\{0\}} \omega^{2_{s}^{*}-2} \phi \psi d x \leq C\|\omega\|_{H_{0, L}^{1}(\mathcal{C})}^{2^{*}-2}\|\phi\|_{H_{0, L}^{1}(\mathcal{C})}\|\psi\|_{H_{0, L}^{1}(\mathcal{C})} .
\end{aligned}
$$

As a result, we complete the proof.

Lemma 2.4 There is a constant $C>0$ independent of $\mu$ such that

$$
\|\ell\|_{H_{0, L}^{1}(\mathcal{C})} \leq C\left(\frac{1}{\mu^{N-2 s}}+\frac{\lambda}{\mu^{2 s}}\right)
$$

Proof Recall

$$
\ell(\omega)=\int_{\Omega \times\{0\}} U_{x, \mu}^{2_{s}^{*}-1} \omega d x-\int_{\Omega \times\{0\}}\left|P_{\Omega} U_{x, \mu}\right|^{2_{s}^{*}-1} \omega d x-\lambda \int_{\Omega \times\{0\}}\left(P_{\Omega} U_{x, \mu}\right) \omega d x
$$

By a direct calculation, we have

$$
\begin{aligned}
\left.|| P_{\Omega} U_{x, \mu}\right|^{2_{s}^{*}-1}-\left|U_{x, \mu}\right|^{2_{s}^{*}-1} \mid & =|| U_{x, \mu}-\left.\varphi\right|^{2_{s}^{*}-1}-\left|U_{x, \mu}\right|^{2_{s}^{*}} \mid \\
& \leq C\left|U_{x, \mu}^{2_{s}^{*}-2} \varphi\right| \leq C \frac{1}{\mu^{\frac{N-2 s}{2}}} H\left(\left(x_{0}, 0\right), x_{0}\right) U_{x, \mu}^{2_{s}^{*}-2} .
\end{aligned}
$$

For $\varphi=U_{x, \mu}-P_{\Omega} U_{x, \mu}$, we have

$$
\begin{aligned}
\int_{\Omega \times\{0\}}\left|P_{\Omega} U_{x, \mu}\right|^{2} d x & =\int_{\Omega \times\{0\}}\left(U_{x, \mu}-\varphi\right)^{2} d x \\
& =\int_{\Omega \times\{0\}}\left|U_{x, \mu}\right|^{2} d x-\int_{\Omega \times\{0\}} 2 U_{x, \mu} \varphi d x+\int_{\Omega \times\{0\}} \varphi^{2} d x .
\end{aligned}
$$

By a direct calculation, we have

$$
\int_{\Omega \times\{0\}} \varphi^{2} d x=\int_{\Omega}\left(\frac{1}{\mu^{\frac{N-2 s}{2}}} H((x, 0), x)\right)^{2} d x=O\left(\frac{1}{\mu^{N-2 s}}\right)
$$

and

$$
\begin{aligned}
\int_{\Omega} U_{x, \mu}^{2} d x & =\int_{\Omega \times\{0\}}\left(\frac{\mu}{1+\mu^{2}\left|y-x_{\lambda}\right|^{2}}\right)^{N-2 s} d y=\int_{\Omega_{\mu}} \mu^{-N}\left(\frac{\mu}{1+|z|^{2}}\right)^{N-2 s} d z \\
& =\frac{C_{0}}{\mu^{2 s}} \int_{\mathbb{R}^{N}} \frac{1}{\left(1+|z|^{2}\right)^{N-2 s}} d z+O\left(\frac{1}{\mu^{2 s}}\right)
\end{aligned}
$$

where $\Omega_{\mu}=\left\{y: \mu^{-1} y=x \in \Omega\right\}$. 
Inserting (2.22) and (2.23) to (2.21), we obtain

$$
\int_{\Omega \times\{0\}}\left|P_{\Omega} U_{x, \mu}\right|^{2} d x \leq \frac{C}{\mu^{2 s}}+O\left(\frac{1}{\mu^{2 s+1}}\right)
$$

Combining (2.19), (2.20) and (2.24), we have

$$
\begin{aligned}
& \|\ell(\omega)\|_{H_{0, L}^{1}(\mathcal{C})} \leq \int_{\Omega \times\{0\}}\left(\left|P_{\Omega} U_{x, \mu}\right|^{2_{s}^{*}-1}-U_{x, \mu}^{2_{s}^{*}-1}\right) \omega d x+\lambda \int_{\Omega \times\{0\}}\left(P_{\Omega} U_{x, \mu}\right) \omega d x \\
& \leq C \int_{\Omega \times\{0\}} \frac{U_{x, \mu}^{2_{s}^{*}-2}}{\mu^{\frac{N-2 s}{2}}} H\left(\left(x_{0}, 0\right), x_{0}\right) \omega d x+\lambda \int_{\Omega \times\{0\}}\left(P_{\Omega} U_{x, \mu}\right) \omega d x \\
& \leq \frac{C}{\mu^{\frac{N-2 s}{2}}}\left(\int_{\Omega \times\{0\}}\left|U_{x, \mu}^{2_{s}^{*}-2} H\left(\left(x_{0}, 0\right), x_{0}\right)\right|^{\frac{2 N}{N+2 s}} d x\right)^{\frac{N+2 s}{2 N}}\|\omega\|_{H_{0, L}^{1}(\mathcal{C})} \\
& +\lambda\left(\int_{\Omega \times\{0\}}\left(P_{\Omega} U_{x, \mu}\right)^{2} d x\right)^{\frac{1}{2}}\|\omega\|_{H_{0, L}^{1}(\mathcal{C})} \\
& \leq \frac{C}{\mu^{\frac{N-2 s}{2}}}\left(\int_{\Omega \times\{0\}}\left|U_{x, \mu}^{2_{s}^{*}-2}\right|^{\frac{2_{s}^{*}}{2_{s}^{*}-2}} d x\right)^{\frac{2_{s}^{*}-2}{2_{s}^{*}}}\|\omega\|_{H_{0, L}^{1}(\mathcal{C})} \\
& +\lambda\left(\int_{\Omega \times\{0\}}\left(P_{\Omega} U_{x, \mu}\right)^{2} d x\right)^{\frac{1}{2}}\|\omega\|_{H_{0, L}^{1}(\mathcal{C})} \\
& \leq C\left(\frac{1}{\mu^{N-2 s}}+\frac{\lambda}{\mu^{2 s}}\right)\|\omega\|_{H_{0, L}^{1}(\mathcal{C})} .
\end{aligned}
$$

Then we get the conclusion.

\section{The finite-dimensional reduction and proof of the main results}

In this section, we intend to prove the main theorem by the Lyapunov-Schmidt reduction. It is easy to check that $L w$ can be generated by a bounded linear operator $L$ from $E$ to $E$, which is defined as

$$
\langle L \omega, \varphi\rangle=\int_{\mathcal{C}} y^{1-2 s} \nabla \omega \nabla \varphi d x d y-\lambda \int_{\Omega \times\{0\}} \omega \varphi d x-\left(2_{s}^{*}-1\right) \int_{\Omega \times\{0\}}\left(P_{\Omega} U_{x, \mu}\right)^{2_{s}^{*}-2} \omega \varphi d x
$$

Next, we show the invertibility of $L$ in $E$.

Proposition 3.1 There exists a constant $\rho>0$, such that

$$
\|P L \omega\|_{H_{0, L}^{1}(\mathcal{C})} \geq \rho\|\omega\|_{H_{0, L}^{1}(\mathcal{C})}, \quad \omega \in E
$$

Proof We argue it by contradiction. Suppose that there are $n \rightarrow+\infty, x_{n} \rightarrow x_{0}, \mu_{n} \rightarrow+\infty$, $\lambda_{n} \rightarrow 0, \omega_{n} \in E$, such that

$$
\left\|P L \omega_{n}\right\|_{H_{0, L}^{1}(\mathcal{C})} \leq \frac{1}{n}\left\|\omega_{n}\right\|_{H_{0, L}^{1}(\mathcal{C})} .
$$

Without loss of generality, we assume $\left\|\omega_{n}\right\|_{H_{0, L}^{1}(\mathcal{C})}=1$, then $\left\|P L \omega_{n}\right\|_{H_{0, L}^{1}(\mathcal{C})} \leq \frac{1}{n}$. 
Then

$$
\begin{gathered}
\int_{\mathcal{C}} y^{1-2 s} \nabla \varphi \nabla \omega_{n} d x d y-\lambda_{n} \int_{\Omega \times\{0\}} \varphi \omega_{n} d x-\left(2_{s}^{*}-1\right) \int_{\Omega \times\{0\}}\left(P_{\Omega} U_{x_{n}, \mu_{n}}\right)^{2_{s}^{*}-2} \varphi \omega_{n} d x \\
=o(1)\|\varphi\|_{H_{0, L}^{1}(\mathcal{C})}+\alpha_{0}\left\langle\frac{\partial P_{\Omega} U_{x_{n}, \mu_{n}}}{\partial \mu}, \varphi\right\rangle_{H_{0, L}^{1}(\mathcal{C})}+\sum_{i=1}^{N} \alpha_{i}\left(\frac{\partial P_{\Omega} U_{x_{n}, \mu_{n}}}{\partial x_{i}}, \varphi\right\rangle_{H_{0, L}^{1}(\mathcal{C})} .
\end{gathered}
$$

Step 1 , we claim $\alpha_{0}, \alpha_{i}=0$ for $(i=1, \ldots, N)$. Let $\varphi=\partial P_{\Omega} U_{x_{n}, \mu_{n}}$, we obtain

$$
\begin{aligned}
\int_{\mathcal{C}} & y^{1-2 s} \nabla\left(\partial P_{\Omega} U_{x_{n}, \mu_{n}}\right) \nabla \omega_{n} d x d y-\lambda_{n} \int_{\Omega \times\{0\}}\left(\partial P_{\Omega} U_{x_{n}, \mu_{n}}\right) \omega_{n} d x \\
& -\left(2_{s}^{*}-1\right) \int_{\Omega \times\{0\}}\left(P_{\Omega} U_{x_{n}, \mu_{n}}\right)^{2_{s}^{*}-2}\left(\partial P_{\Omega} U_{x_{n}, \mu_{n}}\right) \omega_{n} d x \\
= & \int_{\Omega \times\{0\}}\left(2_{s}^{*}-1\right) U_{x_{n}, \mu_{n}}^{2_{s}^{*}-2} \partial\left(P_{\Omega} U_{x_{n}, \mu_{n}}\right) \omega_{n} d x-\lambda_{n} \int_{\Omega \times\{0\}} \partial\left(P_{\Omega} U_{x_{n}, \mu_{n}}\right) \omega_{n} d x \\
& -\left(2_{s}^{*}-1\right) \int_{\Omega \times\{0\}}\left(P_{\Omega} U_{x_{n}, \mu_{n}}\right)^{2_{s}^{*}-2} \partial\left(P_{\Omega} U_{x_{n}, \mu_{n}}\right) \omega_{n} d x \\
= & o_{n}(1),
\end{aligned}
$$

where $\partial\left(P_{\Omega} U_{x_{n}, \mu_{n}}\right)=\frac{\partial\left(P_{\Omega} U_{x_{n}, \mu_{n}}\right)}{\partial \mu}$ or $\frac{\partial\left(P_{\Omega} U_{x_{n}, \mu_{n}}\right)}{\partial x_{i}}$ for $i=1, \ldots, N$. Then $\alpha_{0}, \alpha_{i}=0$ for $(i=$ $1, \ldots, N)$.

Step 2, we show that $\int_{\Omega \times\{0\}}\left(P_{\Omega} U_{x_{n}, \mu_{n}}\right)^{2_{s}^{*}-2} \partial\left(P_{\Omega} U_{x_{n}, \mu_{n}}\right) \omega_{n} d x=o_{n}(1)$, since

$$
\begin{aligned}
& \int_{\Omega \times\{0\}}\left|P_{\Omega} U_{x_{n}, \mu_{n}}\right|^{2_{s}^{*}-2} \partial\left(P_{\Omega} U_{x_{n}, \mu_{n}}\right) \omega_{n} d x \\
& \quad=\left(\int_{\left\{\left(\mu_{n}\left|y-x_{n}\right| \geq R\right) \times\{0\}\right\}}+\int_{\left\{\left(\mu_{n}\left|y-x_{n}\right| \leq R\right) \times\{0\}\right\}}\right)\left|P_{\Omega} U_{x_{n}, \mu_{n}}\right|^{2_{s}^{*}-2} \partial\left(P_{\Omega} U_{x_{n}, \mu_{n}}\right) \omega_{n} d x .
\end{aligned}
$$

We consider the following inequality:

$$
\begin{aligned}
& \int_{\left\{\left(\mu_{n}\left|y-x_{n}\right| \geq R\right) \times\{0\}\right\}}\left|\left(P_{\Omega} U_{x_{n}, \mu_{n}}\right)^{2_{s}^{*}-2}\left(\partial P_{\Omega} U_{x_{n}, \mu_{n}}\right) \omega_{n}\right| d x \\
& \quad \leq \int_{\left(\mu_{n}\left|y-x_{n}\right| \geq R\right) \times\{0\}}\left|U_{x_{n}, \mu_{n}}\right|_{s}^{2_{s}^{*}-2} \partial U_{x_{n}, \mu_{n}} \omega_{n} d x \\
& \quad=\int_{\{(|z| \geq R) \times\{0\}\}} U_{0,1}^{2_{s}^{*}-2} \partial U_{0,1} \tilde{\omega}_{n} d x \\
& \quad \leq\left(\int_{\{(|z| \geq R) \times\{0\}\}}\left(U_{0,1}^{2_{s}^{*}-2} \partial U_{0,1}\right)^{\frac{2 N}{N+2 s}} d x\right)^{\frac{N+2 s}{2 N}}\left\|\tilde{\omega}_{n}\right\|_{L^{2_{s}^{*}}(\Omega)} \\
& \quad=o_{R}(1)\|\omega\|_{L^{2}(\Omega)}=o_{R}(1),
\end{aligned}
$$

where $\tilde{\omega}_{n}(y)=\mu_{n}^{-\frac{N-2 s}{2}} \omega\left(\mu_{n}^{-1} x+x_{n}\right), z=\mu_{n}\left|y-x_{n}\right|$. 
Now, we consider $\int_{\left\{\left(\mu_{n}\left|y-x_{n}\right| \leq R\right) \times\{0\}\right\}}\left|P_{\Omega} U_{x_{n}, \mu_{n}}\right|^{2_{s}^{*}-2} \partial\left(P_{\Omega} U_{x_{n}, \mu_{n}}\right) \omega_{n} d x$. Since $\left\|\omega_{n}(x)\right\|_{H_{0, L}^{1}(\mathcal{C})}=1$, then $\left\|\tilde{\omega}_{n}\right\|_{H_{0, L}^{1}(\mathcal{C})}=1$. Then $\left\{\tilde{w}_{n}\right\}$ is bounded in $H_{0, L}^{1}(\mathcal{C})$. We have

$$
\begin{aligned}
& \tilde{\omega}_{n} \rightarrow \omega, \quad \text { weakly in } H_{0, L}^{1}(\mathcal{C}), \\
& \tilde{\omega}_{n}(x, 0) \rightarrow \omega(x, 0), \quad \text { strongly in } L^{p}(\Omega)\left(1<p<2_{s}^{*}\right) .
\end{aligned}
$$

Since $\tilde{\omega}_{n}(y)$ satisfies

$$
\begin{cases}\operatorname{div}\left(t^{1-2 s} \nabla \tilde{\omega}_{n}\right)=0, & \text { in } \Omega_{\mu} \times\{(0,+\infty)\}, \\ \tilde{\omega}_{n}=0, & \text { on } \partial_{L} \Omega_{\mu} \times\{(0,+\infty)\}, \\ \partial_{v}^{s} \tilde{\omega}_{n}=\tilde{\omega}_{n}^{2_{s}^{*}-1}+\lambda_{n} \tilde{\omega}_{n}, & \text { in } \Omega_{\mu} \times\{0\},\end{cases}
$$

we see that $\omega$ satisfies the following equations:

$$
\begin{cases}\operatorname{div}\left(t^{1-2 s} \nabla \omega\right)=0, & \text { in } \mathbb{R}_{+}^{N+1}, \\ \omega>0, & \text { in } \mathbb{R}_{+}^{N+1}, \\ \partial_{v}^{s} \omega=\omega^{2_{s}^{*}-1}, & \text { in } \mathbb{R}^{N} \times\{0\}\end{cases}
$$

hence $\omega=\alpha_{0} \frac{\partial U_{0,1}}{\partial \mu}+\sum_{i=1}^{N} \alpha_{i} \frac{\partial U_{0,1}}{\partial x_{i}}$.

Since $\omega_{n} \in E$, then $\left\langle w_{n}, \frac{\partial U_{x_{n}, \mu_{n}}}{\partial \mu}\right\rangle_{\left(H_{0, L}^{s}(\mathcal{C})\right)}=0,\left\langle\omega_{n}, \frac{\partial U_{x_{n}, \mu_{n}}}{\partial x_{i}}\right\rangle_{H_{0, L}^{1}(\mathcal{C})}=0$, then we get $\left\langle\omega, \partial U_{0,1}\right\rangle_{H_{0, L}^{1}(\mathcal{C})}=0$. Thus $\omega=0$ and

$$
\int_{\left\{\left(\mu_{n}\left|y-x_{n}\right| \leq R\right) \times\{0\}\right\}}\left|P_{\Omega} U_{x_{n}, \mu_{n}}\right|^{2_{s}^{*}-2} \partial\left(P_{\Omega} U_{x_{n}, \mu_{n}}\right) \omega_{n} d x \leq C\left(\int_{|y| \leq R} \omega_{n}^{2}\right)^{\frac{1}{2}} \rightarrow 0 .
$$

Combining (3.3) and (3.7), we obtain $\int_{\Omega \times\{0\}}\left(P_{\Omega} U_{x_{n}, \mu_{n}}\right)^{2_{s}^{*}-2} \partial\left(P_{\Omega} U_{x_{n}, \mu_{n}}\right) \omega_{n} d x=o_{n}(1)$.

Step 3 , in (3.1), we denote $\varphi=w_{n}$, we have

$$
\begin{aligned}
o_{n}(1)\left\|w_{n}\right\|_{H_{0, L}^{1}(\mathcal{C})}= & \int_{\mathcal{C}} y^{1-2 s}\left|\nabla \omega_{n}\right|^{2} d x d y-\lambda_{n} \int_{\Omega \times\{0\}}\left|\omega_{n}\right|^{2} d x \\
& -\left(2_{s}^{*}-1\right) \int_{\Omega \times\{0\}}\left(P_{\Omega} U_{x_{n}, \mu_{n}}\right)^{2_{s}^{*}-2}\left|\omega_{n}\right|^{2} d x \\
= & \left\|\omega_{n}\right\|_{H_{0, L}^{1}(\mathcal{C})}^{2}+o_{n}(1)\left\|\omega_{n}\right\|_{H_{0, L}^{1}(\mathcal{C})}^{2},
\end{aligned}
$$

we get a contradiction, thus $L$ is invertible.

Now we perform the finite-dimensional reduction procedure.

Proposition 3.2 There is a $C^{1}$ map from $S$ to $H_{0, L}^{1}: \omega=\omega(\mu)$, satisfying $\omega \in E$, and

$$
\left.J^{\prime}(\omega)\right|_{E}=0
$$

Moreover, there exists a constant $C>0$ independent of $\mu$ such that

$$
\|\omega\|_{H_{0, L}^{1}(\mathcal{C})} \leq C\left(\frac{1}{\mu^{N-2 s}}+\frac{\lambda}{\mu^{2 s}}\right) .
$$


Proof We will use the contraction theorem to prove it. It is known that $\ell(\omega)$ is a bounded linear functional in $E$. By the Riesz representation theorem, there is an $\ell \in E$ such that

$$
\ell(\omega)=\langle\ell, \omega\rangle
$$

So, finding a critical point for $I(\omega)$ is equivalent to solving

$$
\ell+L \omega+R^{\prime}(\omega)=0
$$

By Proposition 3.1, $L$ is invertible. Thus (3.11) is equivalent to

$$
\omega=A(\omega):=-L^{-1}\left(\ell+R^{\prime}(\omega)\right) .
$$

Set

$$
S:=\left\{\omega \in E:\|\omega\|_{H_{0, L}^{1}(\mathcal{C})} \leq\left(\frac{C}{\mu^{N-2 s}}+\frac{\lambda}{\mu^{2 s}}\right)^{1-\theta}\right\} .
$$

We shall verify that $A$ is a contraction mapping from $S$ to itself. In fact, on the one hand, for any $\omega \in S$, we obtain

$$
\begin{aligned}
\|A(\omega)\|_{H_{0, L}^{1}(\mathcal{C})} & \leq C\left(\|\ell\|_{H_{0, L}^{1}(\mathcal{C})}+\left\|R^{\prime}(\omega)\right\|_{H_{0, L}^{1}(\mathcal{C})}\right) \\
& \leq C\left(\|\ell\|_{H_{0, L}^{1}(\mathcal{C})}+\|\omega\|_{H_{0, L}^{1}(\mathcal{C})}^{\min \left\{2^{*}-1,2\right\}}\right) \\
& \leq\left(\frac{C}{\mu^{N-2 s}}+\frac{\lambda}{\mu^{2 s}}\right)+\left(\frac{C}{\mu^{N-2 s}}+\frac{\lambda}{\mu^{2 s}}\right)^{(1-\theta) \min \left\{2_{s}^{*}-1,2\right\}} \\
& \leq \frac{C}{\mu^{N-2 s}}+\frac{\lambda}{\mu^{2 s}} .
\end{aligned}
$$

On the other hand, for any $\omega_{1}, \omega_{2} \in S$,

$$
\begin{aligned}
\left\|A\left(\omega_{1}\right)-A\left(\omega_{2}\right)\right\|_{H_{0, L}^{1}(\mathcal{C})} & =\left\|L^{-1} R^{\prime}\left(\omega_{1}\right)-L^{-1} R^{\prime}\left(\omega_{2}\right)\right\|_{H_{0, L}^{1}(\mathcal{C})} \\
& \leq C\left\|R^{\prime}\left(\omega_{1}\right)-R^{\prime}\left(\omega_{2}\right)\right\|_{H_{0, L}^{1}(\mathcal{C})} \\
& \leq C\left\|R^{\prime \prime}\left(\theta \omega_{1}+(1-\theta) \omega_{2}\right)\right\|\left\|\omega_{1}-\omega_{2}\right\|_{H_{0, L}^{1}(\mathcal{C})} \\
& \leq C\left\|\theta \omega_{1}+(1-\theta) \omega_{2}\right\|_{H_{0, L}^{1}(\mathcal{C})}^{\min \left\{2^{*}-2,1\right\}}\left\|\omega_{1}-\omega_{2}\right\|_{H_{0, L}^{1}(\mathcal{C})} \\
& \leq \frac{1}{2}\left\|\omega_{1}-\omega_{2}\right\|_{H_{0, L}^{1}(\mathcal{C})} .
\end{aligned}
$$

Then the result follows from the contraction mapping theorem. 
We proved that there exist $\alpha_{i}(i=0,1, \ldots, j)$ satisfying

$$
\begin{aligned}
& (-\Delta)^{S}\left(P_{\Omega} U_{x, \mu}+\omega\right)-\lambda\left(P_{\Omega} U_{x, \mu}+\omega\right)-\left(P_{\Omega} U_{x, \mu}+\omega\right)^{2_{s}^{*}-1} \\
& =\alpha_{0} \frac{\partial P_{\Omega} U_{x, \mu}}{\partial \mu}+\sum_{i=1}^{N} \alpha_{i} \frac{\partial P_{\Omega} U_{x, \mu}}{\partial x_{i}}
\end{aligned}
$$

and $\|\omega\|_{H_{0, L}^{1}(\mathcal{C})} \leq \frac{C}{\mu^{N-2 s}}+C \frac{\lambda}{\mu^{2}}$.

Now, we want to show that $\alpha_{0}=\alpha_{1}=\cdots=\alpha_{n}=0$. First we denote

$$
\begin{aligned}
\omega(x, \mu)= & \frac{1}{2} \int_{\mathcal{C}} y^{1-2 s}\left|\nabla\left(P_{\Omega} U_{x, \mu}+\omega\right)\right|^{2} d x d y-\frac{\lambda}{2} \int_{\Omega \times\{0\}}\left|P_{\Omega} U_{x, \mu}+\omega\right|^{2} d x \\
& -\frac{1}{2_{s}^{*}} \int_{\Omega \times\{0\}}\left|P_{\Omega} U_{x, \mu}+\omega\right|^{2_{s}^{*}} d x .
\end{aligned}
$$

Next, we have the following lemma.

Lemma 3.3 If $(x, \mu)$ is the critical point of $\omega(x, \mu)$, then $\alpha_{0}=\alpha_{1}=\cdots=\alpha_{n}=0$.

Proof We can refer to [19], we omit the proof.

Now, we consider the critical point of $w(x, \mu)$. For $\omega=0$, we obtain

$$
\begin{aligned}
\bar{\omega}(x, \mu)= & \frac{1}{2 C_{s}} \int_{\mathcal{C}} y^{1-2 s}\left|\nabla P_{\Omega} U_{x, \mu}\right|^{2} d x d y-\frac{\lambda}{2} \int_{\Omega \times\{0\}}\left|P_{\Omega} U_{x, \mu}\right|^{2} d x \\
& -\frac{1}{2_{s}^{*}} \int_{\Omega \times\{0\}}\left|P_{\Omega} U_{x, \mu}\right|^{2_{s}^{*}} d x \\
= & \frac{s}{N} \int_{\mathbb{R}^{N}} u_{0,1}^{2_{s}^{*}} d x+\frac{B_{0} H((x, 0), x)}{\mu^{N-2 s}}-\frac{\lambda B_{1}}{\mu^{2}}+\text { h.o.t, }
\end{aligned}
$$

where $B_{0}, B_{1}$ are defined in the appendix.

By a direct calculation, we get

$$
\begin{aligned}
\omega(x, \mu)= & \bar{\omega}_{x, \mu}+\int_{\mathcal{C}} \nabla\left(P_{\Omega} U_{x, \mu}\right) \nabla \omega d x d y \\
& -\lambda \int_{\Omega \times\{0\}}\left(P_{\Omega} U_{x, \mu}\right) \omega d x-\int_{\Omega \times\{0\}}\left(P_{\Omega} U_{x, \mu}\right)^{2_{s}^{*}-1} \omega d x \\
& -\frac{1}{2_{s}^{*}} \int_{\Omega \times\{0\}}\left(P_{\Omega} U_{x, \mu}+\omega\right)^{2_{s}^{*}}-\left(P_{\Omega} U_{x, \mu}\right)^{2_{s}^{*}}-2_{s}^{*}\left(P_{\Omega} U_{x, \mu}\right)^{2_{s}^{*}-1} \omega d x \\
& +\frac{1}{2} \int_{\mathcal{C}}|\nabla \omega|^{2} d x d y-\frac{1}{2} \lambda \int_{\Omega \times\{0\}} \omega^{2} d x .
\end{aligned}
$$


Since $\|\omega\|_{H_{0, L}^{1}(\mathcal{C})} \leq C\left(\frac{1}{\mu^{N-2 s}}+\frac{\lambda}{\mu^{2}}\right)$, we have

$$
\begin{aligned}
\int_{\mathcal{C}} & \nabla\left(P_{\Omega} U_{x, \mu}\right) \nabla \omega d x d y-\lambda \int_{\Omega \times\{0\}}\left(P_{\Omega} U_{x, \mu}\right) \omega d x-\int_{\Omega \times\{0\}}\left(P_{\Omega} U_{x, \mu}\right)^{2_{s}^{*}-1} \omega d x \\
= & \int_{\Omega \times\{0\}} U_{x, \mu}^{2_{s}^{*}-1} \omega d x d y-\lambda \int_{\Omega \times\{0\}}\left(P_{\Omega} U_{x, \mu}\right) \omega d x-\int_{\Omega \times\{0\}}\left(P_{\Omega} U_{x, \mu}\right)^{2_{s}^{*}-1} \omega d x \\
& =o(1)\|\omega\|_{H_{0, L}^{1}(\mathcal{C})} .
\end{aligned}
$$

Combining (3.15) and (3.16), we obtain

$$
\begin{aligned}
\omega(x, \mu) & =\bar{\omega}(x, \mu)+o(1)\|\omega\|_{H_{0, L}^{1}(\mathcal{C})} \\
& =A+\frac{B_{0} H((x, 0), x)}{\mu^{N-2 s}}-\frac{\lambda B_{1}}{\mu^{2}}+o\left(\frac{1}{\mu^{N-2 s}}+\frac{\lambda}{\mu^{2}}\right) .
\end{aligned}
$$

Then we get $\left(x_{0}, \mu_{0}\right)$ is the critical point of $\omega(x, \mu)$, where $x_{0}$ is the local minimizer of the Robin function $H\left(\left(x_{0}, 0\right), x_{0}\right)$, and $\mu=\mu_{0}=\left(\frac{(N-2 s) B_{0} H\left(\left(x_{0}, 0\right), x_{0}\right)}{2 \lambda B_{1}}\right) \frac{1}{N-2 s-4}$.

\section{Appendix: Energy expansion}

In this section, we will give the energy expansion for the approximate solution. Recall

$$
I(v)=\frac{1}{2} \int_{\mathcal{C}} y^{1-2 s}|\nabla v|^{2} d x d y-\frac{\lambda}{2} \int_{\Omega \times\{0\}}|v|^{2} d x-\frac{1}{p+1} \int_{\Omega \times\{0\}}|v|^{p+1} d x
$$

Proposition A.1 We have

$$
I\left(P_{\Omega} U_{x, \mu}\right)=A+\frac{B}{\mu^{N-2 s}} H\left(\left(x_{0}, 0\right), x_{0}\right)-\frac{\lambda B_{1}}{\mu^{2 s}}+O\left(\frac{1}{\mu^{N-2 s+1}}\right),
$$

where $A=C_{1} \int_{\mathbb{R}^{N}} u_{0,1}^{2_{s}^{*}} d x, B=C_{2} \int_{\mathbb{R}^{N}} \frac{1}{\left(1+|z|^{2}\right)^{\frac{N+2 s}{2}}} d z$ and $B_{1}=C_{0} \int_{\mathbb{R}^{N}} \frac{1}{\left(1+|z|^{2}\right)^{N-2 s}} d z$.

Proof Recall

$$
\begin{aligned}
I\left(P_{\Omega} U_{x, \mu}\right)= & \frac{1}{2} \int_{\mathcal{C}} y^{1-2 s}\left|\nabla P_{\Omega} U_{x, \mu}\right|^{2} d x d y-\frac{\lambda}{2} \int_{\Omega \times\{0\}}\left|P_{\Omega} U_{x, \mu}\right|^{2} d x \\
& -\frac{1}{p+1} \int_{\Omega \times\{0\}}\left|P_{\Omega} U_{x, \mu}\right|^{p+1} d x .
\end{aligned}
$$

First, using (2.11), we note the following identity:

$$
\begin{aligned}
\int_{\mathcal{C}} y^{1-2 s}\left|\nabla\left(P_{\Omega} U_{x, \mu}\right)\right|^{2} d x d y & =\int_{\Omega \times\{0\}} \partial_{v}^{s}\left(P_{\Omega} U_{x, \mu}\right)\left(P_{\Omega} U_{x, \mu}\right) d x \\
& =\int_{\Omega \times\{0\}} U_{x, \mu}^{2_{s}^{*}-1}\left(P_{\Omega} U_{x, \mu}\right) d x \\
& =\int_{\Omega \times\{0\}} U_{x, \mu}^{2_{s}^{*}-1}\left(U_{x, \mu}-\varphi\right) d x
\end{aligned}
$$


Wang Boundary Value Problems

(2020) 2020:145

Page 16 of 18

By a direct computation, we have

$$
\begin{aligned}
\int_{\Omega \times\{0\}} U_{x, \mu}^{2_{s}^{*}} d x & =\int_{\Omega} a_{N, s}^{\frac{2 N}{N-2 s}}\left(\frac{\mu}{1+\mu^{2}\left|x-x_{\lambda}\right|^{2}}\right)^{N} d x \\
& =\int_{\Omega_{\mu}} a_{N, s}^{\frac{2 N}{N-2 s}}\left(\frac{\mu}{1+|z|^{2}}\right)^{N} \mu^{-N} d z=\int_{\Omega_{\mu}} \frac{C_{0}}{\left(1+|z|^{2}\right)^{N}} d z \\
& =\int_{\mathbb{R}^{N}} \frac{C_{0}}{\left(1+|z|^{2}\right)^{N}} d z-\int_{\mathbb{R}^{N} \backslash \Omega_{\mu}} \frac{C_{0}}{\left(1+|z|^{2}\right)^{N}} d z \\
& =\int_{\mathbb{R}^{N}} u_{0,1}^{2_{s}^{*}} d x+O\left(\frac{1}{\mu^{N}}\right)
\end{aligned}
$$

and

$$
\begin{aligned}
& \int_{\Omega \times\{0\}} U_{x, \mu}^{2_{s}^{*}-1} \varphi d x \\
& =\int_{\Omega}\left(a_{N, s}\left(\frac{\mu}{1+\mu^{2}\left|x-x_{\lambda}\right|^{2}}\right)^{\frac{N-2 s}{2}}\right)^{2_{s}^{*-1}}\left(\frac{C_{0}}{\mu^{\frac{N-2 s}{2}}} H((x, 0), y)+\frac{1}{\mu^{\frac{N+2 s}{2}}}\right) d x \\
& =\frac{1}{\mu^{\frac{N-2 s}{2}}} \int_{\Omega}\left(\left(\frac{\mu}{1+\mu^{2}\left|x-x_{\lambda}\right|^{2}}\right)^{\frac{N-2 s}{2}}\right)^{2_{s}^{*}-1} H\left((x, 0), x_{0}\right) d x+O\left(\frac{1}{\mu^{N-2 s+1}}\right) \\
& =\frac{1}{\mu^{\frac{N-2 s}{2}}} \int_{\Omega_{\mu}}\left[\frac{\mu^{\frac{N-2 s}{2}}}{\left(1+|z|^{2}\right)^{\frac{N-2 s}{2}}}\right]^{\frac{N+2 s}{N-2 s}} H\left(\left(\mu^{-1} z+x_{0}\right), x_{0}\right) \mu^{-N} d z+O\left(\frac{1}{\mu^{N-2 s+1}}\right) \\
& =\frac{1}{\mu^{N-2 s}} \int_{\Omega_{\mu}} \frac{H\left(\left(x_{0}, 0\right), x_{0}\right)}{\left(1+|z|^{2}\right)^{\frac{N+2 s}{2}}} d z+O\left(\frac{1}{\mu^{N-2 s+1}}\right) \\
& =\frac{H\left(\left(x_{0}, 0\right), x_{0}\right)}{\mu^{N-2 s}} \int_{\mathbb{R}^{N}} \frac{1}{\left(1+|z|^{2}\right)^{\frac{N+2 s}{2}}} d z+O\left(\frac{1}{\mu^{N-2 s+1}}\right) .
\end{aligned}
$$

Combining (A.4)-(A.6), we obtain

$$
\begin{aligned}
\int_{\mathcal{C}} y^{1-2 s}\left|\nabla\left(P_{\Omega} U_{x, \mu}\right)\right|^{2} d x d y \\
=C_{1} \int_{\mathbb{R}^{N}} u_{0,1}^{2_{s}^{*}} d x-C_{2} \frac{H\left(\left(x_{0}, 0\right), x_{0}\right)}{\mu^{N-2 s}} \int_{\mathbb{R}^{N}} \frac{1}{\left(1+|z|^{2}\right)^{\frac{N+2 s}{2}}} d z+O\left(\frac{1}{\mu^{\frac{N}{2}}}\right) \\
=A-\frac{H\left(\left(x_{0}, 0\right), x_{0}\right)}{\mu^{N-2 s}} B+O\left(\frac{1}{\mu^{\frac{N}{2}}}\right),
\end{aligned}
$$

where $A=C_{1} \int_{\mathbb{R}^{N}} u_{0,1}^{2_{s}^{*}} d x, B=C_{2} \int_{\mathbb{R}^{N}} \frac{1}{\left(1+|z|^{2}\right)^{\frac{N+2 s}{2}}} d z$.

For the second term of the right hand side of (A.3), similarly, we have

$$
\begin{aligned}
\int_{\Omega \times\{0\}}\left|P_{\Omega} U_{x, \mu}\right|^{2_{s}^{*}} d x & =\int_{\Omega \times\{0\}}\left|U_{x, \mu}-\varphi\right|^{2_{s}^{*}} d x \\
& =\int_{\Omega \times\{0\}} U_{x, \mu}^{2_{s}^{*}} d x-2_{s}^{*} \int_{\Omega \times\{0\}} U_{x, \mu}^{2_{s}^{*}-1} \varphi d x+O\left(\int_{\Omega \times\{0\}} U_{x, \mu}^{2_{s}^{*}-2} \varphi^{2}\right) \\
& =A-\frac{2_{s}^{*} H\left(\left(x_{0}, 0\right), x_{0}\right)}{\mu^{N-2 s}} B+O\left(\frac{1}{\mu^{\frac{N-2 s+2}{2}}}\right) .
\end{aligned}
$$


Since $\varphi=U_{x, \mu}-P_{\Omega} U_{x, \mu}$, by a direct computation, we have

$$
\begin{aligned}
\int_{\Omega \times\{0\}}\left|P_{\Omega} U_{x, \mu}\right|^{2} d x & =\int_{\Omega \times\{0\}}\left(U_{x, \mu}-\varphi\right)^{2} d x \\
& =\int_{\Omega \times\{0\}}\left(\left|U_{x, \mu}\right|^{2} d x-\int_{\Omega \times\{0\}} 2 U_{x, \mu} \varphi d x+\int_{\Omega \times\{0\}} \varphi^{2} d x .\right.
\end{aligned}
$$

Since

$$
\int_{\Omega \times\{0\}} \varphi^{2} d x=\int_{\Omega}\left(\frac{1}{\mu^{\frac{N-2 s}{2}}} H((x, 0), x)\right)^{2} d x=O\left(\frac{1}{\mu^{N-2 s}}\right)
$$

and

$$
\begin{aligned}
\int_{\Omega \times\{0\}} U_{x, \mu}^{2} d x & =\int_{\Omega \times\{0\}}\left(\frac{\mu}{1+\mu^{2}|y-x|^{2}}\right)^{N-2 s} d y=\int_{\Omega_{\mu}} \mu^{-N}\left(\frac{\mu}{1+|z|^{2}}\right)^{N-2 s} d z \\
& =\frac{C_{0}}{\mu^{2 s}} \int_{\mathbb{R}^{N}} \frac{1}{\left(1+|z|^{2}\right)^{N-2 s}} d z+O\left(\frac{1}{\mu^{2 s}}\right),
\end{aligned}
$$

where $B_{1}=C_{0} \int_{\mathbb{R}^{N}} \frac{1}{\left(1+|z|^{2}\right)^{N-2 s}} d z$, we obtain

$$
\int_{\Omega \times\{0\}}\left|P_{\Omega} U_{x, \mu}\right|^{2} d x=\frac{B_{1}}{\mu^{2 s}}+O\left(\frac{1}{\mu^{N-2 s}}\right) .
$$

Combining (A.7), (A.8) and (A.12), we get (A.2).

\section{Acknowledgements}

The author thanks the referees for thoughtful reading of the paper and nice suggestions to improve the results.

Funding

This work is supported by the National Natural Science Foundation of China (11701439).

Availability of data and materials

Date sharing not application to this article as no date sets were generated or analyzed during the current study.

\section{Competing interests}

The authors declare that they have no competing interests.

\section{Authors' contributions}

All authors read and approved the final manuscript.

\section{Publisher's Note}

Springer Nature remains neutral with regard to jurisdictional claims in published maps and institutional affiliations.

Received: 11 May 2020 Accepted: 20 August 2020 Published online: 01 September 2020

\section{References}

1. Atkinson, F.V., Brezis, H., Peletier, L.A.: Nodal solutions of elliptic equations with critical Sobolev exponent. J. Differ. Equ. $85,151-170(1990)$

2. Barrios, B., Colorado, E., de Pablo, A., Sanchez, U.: On some critical problems for the fractional Laplacian operator. J. Differ. Equ. 252, 6133-6162 (2012)

3. Brandle, C., Colorado, E., de Pablo, A.: A concave-convex elliptic problem involving the fractional Laplacian. Proc. R. Soc. Edinb. A 143, 39-71 (2013)

4. Brezis, H., Nirenberg, L.: Positive solutions of nonlinear elliptic equations involving critical Sobolev exponent. Commun. Pure Appl. Math. 36, 437-478 (1983)

5. Cábre, X., Sire, J.: Nonlinear equation for fractional Laplacians, I: regularity, maximum principles, and Hamiltonian estimates. Ann. Inst. Henri Poincaré, Anal. Non Linéaire 31, 23-53 (2014) 
6. Cábre, X., Tan, J.: Positive solutions of nonlinear problems involving the square root of the Laplacian. Adv. Math. 224, 2052-2093 (2010)

7. Caffarelli, L., Salsa, S., Silvestre, L.: Regularity estimates for the solution and the free boundary of the obstacle problem for the fractional Laplacian. Invent. Math. 171, 425-461 (2008)

8. Caffarelli, L., Silvestre, L.: An extension problem related to the fractional Laplacian. Commun. Partial Differ. Equ. 32 $1245-1260(2007)$

9. Choi, W., Kim, S., Lee, K.: Asymptotic behavior of solutions for nonlinear elliptic problems with the fractional Laplacian. J. Funct. Anal. 266, 6531-6598 (2014)

10. Davial, J., del Pino, M., Sire, Y.: Nondegeneracy of the bubble in the critical case for non local equations. Proc. Am. Math. Soc. 141, 3865-3870 (2013)

11. Devillanova, G., Solimini, S.: Concentration estimates and multiple solutions to elliptic problem at critical growth. Adv. Differ. Equ. 7, 1257-1280 (2002)

12. Di Nezza, E., Palatucci, G., Valdinoci, E.: Hithiker's guide to the fractional Sobolev spaces. Bull. Sci. Math. 136, 521-573 (2012)

13. Dipierro, S., Palatucci, G., Valdinoci, E.: Existence and symmetry results for a Schrödinger type problem involving the fractional Laplacian. Matematiche 68, 201-216 (2013)

14. Fiscella, A.: A fractional Kirchhoff problem involving a singular term and a critical nonlinearity. Adv. Nonlinear Anal. 8, 645-660 (2019)

15. Ji, C., Fang, F., Zhang, B.: A multiplicity result for asympototically linear Kirchhoff equations. Adv. Nonlinear Anal. 8 , 267-277 (2019)

16. Lions, P.L.: The concentration-compactness principle in the calculus of variations: the limit case. Rev. Mat. Iberoam. 1 , 45-121 (1985)

17. Mingqi, X., Rădulescu, V.D., Zhang, B.: Fractional Kirchhoff problems with critical Trudinger-Morse nonlinearity. Calc. Var. Partial Differ. Equ. 58, 57 (2019)

18. Mingqi, X., Rădulescu, V.D., Zhang, B.: Nonlocal Kirchhoff problems with singular exponential nonlinearity. Appl. Math Optim. (2020). https://doi.org/10.1007/s00245-020-09666-3

19. Musso, M., Pistoia, A.: Multispike solutions for a nonlinear elliptic problem involving the critical Sobolev exponent. Indiana Univ. Math. J. 51, 541-579 (2002)

20. Rey, O.: The role of the Green's function in a nonlinear elliptic equation involving the critical Sobolev exponent. J. Funct. Anal. 89, 1-52 (1990)

21. Servadei, R., Valdinoci, E.: On the spectrum of two different fractional operators. Proc. R. Soc. Edinb. A 144, 831-855 (2014)

22. Silvestre, L.: Regularity of the obstacle problem for a fractional power of the Laplace operator. Commun. Pure Appl. Math. 60, 67-112 (2007)

23. Sire, Y., Valdinoci, E.: Fractional Laplacian phase transitions and boundary reactions: a geometric inequality and a symmetry result. J. Funct. Anal. 256, 1842-1864 (2009)

24. Struwe, M.: A global compactness result for elliptic boundary value problems involving limiting nonlinearities. Math Z. $187,511-517(1984)$

25. Tan, J.: The Brezis-Nirenberg type problem involving the square root of the Laplacian. Calc. Var. Partial Differ. Equ. 42, 21-41 (2011)

26. Tan, J.: Positive solutions for non local elliptic problems. Discrete Contin. Dyn. Syst. 33, 837-859 (2013)

27. Wang, Z., Zhou, H.-S.: Radial sign-changing solution for fractional Schrödinger equation. Discrete Contin. Dyn. Syst. $36,499-508(2016)$

28. Weinstein, M.: Existence and dynamic stability of solitary wave solutions of equations arising in long wave propagation. Commun. Partial Differ. Equ. 12, 1133-1173 (1987)

29. Xiang, M., Zhang, B., Rădulescu, V.D.: Superlinear Schrödinger-Kirchhoff type problems involving the fractional p-Laplacian and critical exponent. Adv. Nonlinear Anal. 9, 690-709 (2020)

30. Yan, S., Yang, J., Yu, X.: Equations involving fractional Laplacian operator: compactness and application. J. Funct. Anal. $269,47-79(2015)$

\section{Submit your manuscript to a SpringerOpen ${ }^{\circ}$ journal and benefit from:}

- Convenient online submission

- Rigorous peer review

- Open access: articles freely available online

- High visibility within the field

- Retaining the copyright to your article

Submit your next manuscript at $\gg$ springeropen.com 